\author{
Ülo Matjus
}

\title{
DIE STADT ALS ERINNERUNG: UNZEITGEMÄSSE NOTIZEN
}

Nachdem ich den Vorschlag des Lehrstuhls für Kunstgeschichte der Universität Tartu angenommen hatte, während der Herbstschule „Diese Stadt und die andere ${ }^{\prime 1}$, in der es um die Stadt- und Baukunst ging, einen Vortrag eigener Wahl zu einem Thema zu halten, das zu dem Themenkreis passte, konnte ich damals, das heißt bei dem Versuch mir noch nicht recht vorstellen, w ov on ich sprechen könnte oder gar müsste. Ich konnte mich nur gut daran erinnern, dass die Rede von ... der Stadt sein sollte, nichts weiter. Nachdem ich später das vorläufige Programm der Veranstaltung erhalten hatte, dem ich die Überschrift meines Vortrages hinzufügen sollte, fand ich dort mehrere Überschriften mit dem Anfang Die Stadt als ... vor, was mich auf Anhieb für die Überschrift Die Stadt als Erinnerung einstimmte. Ich bezeuge, dass ich dabei einzig über einige verstreute Gedanken verfügte, aber über gute Ideen, die den Vortrag als „,roter Faden“ durchziehen oder als Schwerpunkt fokussieren hätten können, verfügte ich eigentlich noch nicht.

Ich fing dann vorsichtshalber an nachzuschauen oder nachzuforschen, was diese Stadt denn eigentlich bedeutet, wie man sie früher verstanden hat. Ich fand unterschiedliche "offizielle" Definitionen-Begriffsbestimmungen, die von Vertretern verschiedener Wissenschaftsgebiete (wie der Geographie, Kartographie, Kunst- und

DOI: http://dx.doi.org/10.12697/BJAH.2013.5.03

1 Übersetzung aus dem Estnischen von Marju und Olaf Mertelsmann.

Grundlage des vorliegenden essayistischen Beitrages ist ein Vortrag, der während der internationalen Herbstschule „Diese Stadt und die andere“ (Tartu, vom 19. bis zum 21. Oktober 2012), organisiert vom kunsthistorischen Lehrstuhl der Universität Tartu, gehalten wurde. 
Architekturwissenschaft, Wirtschaftswissenschaft, Statistik usw.) stammten, aber sie befriedigten mich nicht. Ich fühlte klar und deutlich, dass allen etwas fehlte, aber was dieses etwas war, das fiel mir schwer in Worte zu fassen - sonst hätte es ja auch keinen Sinn gehabt, die vorhandenen „Definitionen“ nachzuschlagen. Ich führe hier eine als Beispiel vor: „Eine große Siedlung mit eigener Verwaltung und juristischen Rechten, wo die Menschen ihren Unterhalt nicht aus der Landwirtschaft beziehen“. ${ }^{2}$ Mir wurde klar, dass das wenigstens für mich eigentlich nichts über die Natur oder das Wesen der Stadt aussagt, es handelt sich um eine typische reallogische Definition, die auf dem Genus- oder dem nächsten Gattungsbegriff (genus proximum) der Siedlung basiert und dem Artunterschiede oder differentia specifica hinzufügt. Einer dieser Artunterschiede ist das „nichtpositive“, ihren Unterhalt nicht aus der Landwirtschaft beziehen. Die Definition erschien am Anfang nicht durchdacht zu sein, obzwar sie offenbar den Anforderungen einer wissenschaftlichen Begriffsbestimmung entspricht. Ich hätte erfahren wollen, was die Stadt ist, nicht was sie nicht ist - dass sie 1) nicht klein ist und 2) ihren Unterhalt nicht aus der Landwirtschaft bezieht. Nachdem ich nachgedacht hatte, begriff ich, dass ich mehr gewollt hatte, als egal welche Definition an sich zu liefern imstande wäre, denn ihre Aufgabe ist irgendetwas zu bestimmen (de + finio, ich begrenze, bestimme) und von anderem in erster Linie durch Angleichung und Vergleich zu differenzieren, als unterschiedlich, nicht "positiv“ zu erkennen. Selbstverständlich kann und sollte man auch die Stadt von weiteren Siedlungen differenzieren, dies genügt aber nicht, um zu verstehen, was sie ist, diese Stadt, um zu begreifen, worum es sich beim Wesen oder der Natur der Stadt handelt.

Um dem zu begegnen, was die Stadt als eine mich "befriedigende" Stadt darstellt, entschied ich mich anders vorzugehen. Nämlich endlich auch selbst danach zu handeln, was ich während der Vorlesungen und Seminare zur Philosophie den anderen versucht habe beizubringen zu vernehmen ${ }^{3}$ und sehen zu lassen ${ }^{4}$. Ich musste mich freilassen, mich von

2 Eesti etümoloogia-sõnaraamat, Chefherausgeberin Iris Metsmägi (Tallinn: Eesti Keele Sihtasutus, 2012), 242; siehe auch: Eesti keele seletav sõnaraamat, Bd. 3: L-O; Herausgeber Margit Langemets, Mai Tiits, Tiia Valdre, Leidi Veskis, Ülle Viks, Piret Voll (Tallinn: Eesti Keele Sihtasutus, 2009), 143. 3 Siehe Martin Heidegger, Sissejuhatus metafüüsikasse, Übersetzer und Autor des Nachwortes Ülo Matjus (Tartu: Ilmamaa, 1996, 1999), 181-186, 210-221; siehe auch Martin Heidegger, Einführung in die Metaphysik, Martin Heidegger, Gesamtausgabe. II. Abteilung: Vorlesungen 1923-1944, Band 40 (Frankfurt am Main: Vittorio Klostermann, 1983), 148-153, 174-183.

4 Siehe Martin Heidegger, Sissejuhatus metafüüsikasse, 131-153; siehe auch Martin Heidegger, Einführung in die Metaphysik, 105-123. 
den vorgegebenen Definitionen-Begriffsbestimmungen befreien oder entbinden, die zumindest von dem Standpunkt meines Strebens als Vorurteile wirkten oder erschienen. Das bedeutet, dass ich mich vor dem Vorgesagten, vor dem vorher Gemeinten hüten musste, unvoreingenommen offen zur Vernehmung und zum Sehen der Sachen selbst werden sollte und mich nicht davon stören lassen durfte, was ich geglaubt habe, selber früher bereits gewusst und richtig gewusst zu haben oder dass andere dies taten. Es handelt sich dabei um eine Voraussetzung und eines der Momente des phänomenologischen Denkens, der phänomenologischen Haltung im weitesten Sinne. Der Richtungsgeber der modernen Phänomenologie, Edmund Husserl hat dies selbst als „Prinzip aller Prinzipien“ in Worte gefasst, dessen Natur mit der Maxime: „Zu den Sachen selbst!“ zusammengefasst werden könnte. Auf den ersten Blick erscheint dies eine leichte Aufgabe zu sein, aber beim Versuch, dem Aufruf Gefolge zu leisten, stellt sich bald heraus, dass die Aufgabe an sich ganz und gar nicht einfach ist, eher sogar ziemlich schwer - sich vor dem früher Gemeinten in Acht zu nehmen und "Sachen" - dieses Etwas, dieses was es auch sei als sich selbst zu vernehmen und es als sich selbst sehen zu lassen.

Und nun hatte ich mir dies zur Aufgabe gemacht - in Bezug auf die Stadt. Der Stadt selbst sagen zu lassen, was und wie sie von ihrer Natur aus ist - und sie zu vernehmen. Zuzulassen, dass sie sich als Stadt zeigt - und sie als Stadt zu sehen. Aber das kann man nicht "abstrakt", losgerissen, bezüglich irgendeiner Stadt im Allgemeinen durchführen - ich und offenbar jeder andere Städter würde dies in Bezug auf die eigene Stadt in individuo et in concreto machen. Bei mir konnte diese eigene Stadt keine beliebige andere Stadt in der Welt als eben diese Stadt Tartu sein, inmitten der wir uns hier heute gemeinsam befinden.

Ich betonte weiter oben, dass man die Stadt selbst vernehmen muss, aber bevor man sie vernimmt und sie sich als sie selbst sehen lässt, kann und muss man an dieser Stelle auch über das Vernehmen bezüglich der Poesie (Kunst) und des Wortes sprechen. Da ich als Philologe der estnischen Sprache angefangen habe, muss ich mich beständig an einen Satz erinnern, der sich für mich sogar irgendwie wie ein Spuk anhört: „Ein Mädchen kam v o m Lande in die Stadt [meine Sperrung - Ü.M.], es wird erzählt, dass sie barfuß gekommen sei. So wird die Geschichte erzählt, hübsch wie ein Märchen, und deshalb braucht dort auch nicht alles der Wahrheit entsprechen." Der erste Satz selbst verbirgt jedoch 
irgendetwas Großes, Unverfälschtes und Wahrhaftiges, gar eine hoffnungs- und erwartungsvolle Aufregung ... So ist einfach nur ein Dichter imstande, verlauten zu lassen und anzufangen. - Ich meine hier unter der Poesie nicht etwas, was ein Verseschmied oder ein Dichterling zustande gebracht hat, sondern große Poesie, die nicht auf die Unterscheidung der sogenannten Prosa, Poesie und des Dramas achtgibt. Zum Beispiel Goethe, dessen „Prosa“" oder auch „Drama“ mit dem Wort Poesie bezeichnet werden kann. - Erst beim Versuch, den vorgetragenen Satz auszulegen, spürst du die Kraft des Gesagten und die Kraftlosigkeit der eigenen Erklärung... Denn große Poesie ist nicht nacherzählbar. - Aber von wem stammt dieser Satz? Mit dieser „alten Geschichte“ fängt ein estnischer Roman an, nämlich „Võõras majas” [deutsch: „Im fremden Haus"] (1940) von Karl Ristikivi, der erst im Jahre 1943 in der zweiten Auflage seinen richtigen Titel „Õige mehe koda“ [deutsch: „Das Haus des rechten Mannes"] zurückerhielt, den die neuen, kommunistischen Machthaber im Jahre 1940 verboten hatten. ${ }^{5}$ In der estnischen Literaturund Romangeschichte sind aus bestimmten Zeiten noch weitere Beispiele zur Unterscheidung vom Land und der Stadt vorhanden, darüber, wie man aus dem Lande in die Stadt kam. Sofort sollte man sich an Oskar Luts mit seinem autobiographischen Werk „Maa ja linn“ [deutsch: „Land und Stadt"] erinnern, das in Klammern über den Untertitel „Kaks muistset lugu“ [deutsch: „Zwei uralte Geschichten“] verfügt. Ebenso erinnert man sich an Anton Hansen Tammsaare, August Jakobson, Hella MurrikWuolijoki, August Gailit, Bernard Kangro usw. Und noch einiges, wenn man auch die überaus spätere „Plattenbaustadt-Literatur“ im Auge behält (Mati Unt, Teet Kallas, Arvo Valton u.a.). Von Rechts wegen ist zum entfernteren Vorgänger dieser späteren Stadt-Literatur auch der autobiographische Roman von Mati Unt, „Hüvasti, kollane kass“ [deutsch: „Lebe wohl, gelber Kater"] (1963) zu zählen, der mit einer Ankunft in der Stadt anfängt. Übrigens, der Autor kam sogar nach Tartu ... aus Linnamäe [deutsch: Stadtberg].

Wenn man sich nun diese Genannten von ihrem Geist her in Erinnerung ruft und vernimmt, dann kommt in ihnen hauptsächlich eine starke Abgrenzung der Stadt vom Lande zum Vorschein, was oftmals in eine Gegensatzbildung untereinander und in die Feindseligkeit der Stadt

5 Siehe Karl Ristikivi, Võõras majas (Tartu: RK Ilukirjandus ja Kunst, 1940), 5; Karl Ristikivi, Õige mehe koda ([Tartu]: Tartu Eesti Kirjastus, 1943), 5. 
übergeht. Alle Hoffnungen, die sich mit der Stadt verbinden, brauchen nicht in Erfüllung zu gehen, die Stadt ist dem Menschenkind gegenüber feindselig und unbarmherzig. Dass die Stadtluft frei macht, diese historische und „internationale“ Metapher kann auch eine "leere“ Metapher bleiben - ohne eine wirkliche Erfüllung in der Stadt Tallinn oder Tartu zu finden. Karl Ristikivi bezeichnet das Tallinn der Tiina Saalem (so hieß das Mädchen) in der ",alten Geschichte“ als „eine große und erbarmungslose Stadt. ${ }^{\prime 6}$ Was ich sagen möchte, ist, dass die Stadt in unserer estnischen "autobiographischen“ schönen Literatur von ihrer Natur her für diejenigen, die in der Stadt ankommen, meistens nur von der Entfernung aus und am Anfang verlockend reizvoll ist, wonach sie bald anfängt, ihre verborgene Feindseligkeit den Angekommenen gegenüber zu enthüllen. Und dieser estnische klassische Roman ist deshalb nicht öffentlich für unaufrichtig gehalten worden, eher für etwas, was die Augen öffnet oder vorwarnt.

Lasst uns nun vorsichtshalber in Bezug auf die Stadt auch noch in Richtung des Wortes Stadt schauen, um auch das zu vernehmen - das heißt wir sollten zusätzlich auch dem Wort zuhören und auf das Wort hören. Zuallererst setze ich erneut „Eesti keele seletav sõnaraamat" [deutsch: „Erläuterndes Wörterbuch der estnischen Sprache“] (2009) ein, welches dies und jenes erläutert, wie, in welchen Bedeutungen das eine oder das andere Wort in der Sprache verwendet wird - nicht das, ob dieses Wort in irgendeinem Sinne richtig oder falsch verwendet wird, sondern einfach das, was dieses Wort bedeuten kann. Die er ste Bedeutung stellte ich weiter oben vor, diese große, ihren Unterhalt nicht von der Landwirtschaft beziehende Siedlung. Zweiten s kann die Stadt in der älteren, historischen Verwendung eine Bauernburg, eine Burg oder Festung, eine Schanze oder ein Schloss bedeuten. ${ }^{7}$ An dieser Stelle hilft uns unterstützend noch "Eesti etümoloogia sõnaraamat" [deutsch: „Estnisches etymologisches Wörterbuch“] (2012) weiter, das hauptsächlich Materialien über den Ursprung des estnischen, aber breit angelegter auch des uralischen und enger des ostseefinnischen Stamm-(Wort)Schatzes zusammenfasst. Stadt gehört zu diesem Stamm-(Wort)-Schatz, indem sie auch bezüglich ihres Ursprungs Festung oder Schanze bedeutet, in anderen ostseefinnischen Sprachen wie auch im Estnischen bedeu-

6 Karl Ristikivi, Võõras majas, 13; Karl Ristikivi, Õige mehe koda, 13.

7 Siehe Eesti keele seletav sõnaraamat, Bd. 3: L-O, 143. 
tet Stadt, ebenso wie im Finnischen und im Livischen, auch Schloss. ${ }^{8}$ Was könnte man von hier entnehmen, wenn man die Worte in ihrem Ursprung vernimmt, ohne sie für konventionelle und beliebige Zeichen zu halten? Nämlich die Tatsache, dass die Stadt ursprünglich, historisch gesehen eine Anlage oder einen Bau bezeichnet hat, die oder der von uns selbst errichtet wurde, um uns selbst, die Menschen, zu beschützen, als ein Zufluchtsort vor einer drohenden Gefahr, vor dem Feind oder Feinden. Bauernburg, Burg oder Festung und Schanze - die Worte sprechen für sich - die Stadt musste uns beschützen und bewahren, uns Sicherheit und Unterschlupf bieten. Die Namen Taanilinn [deutsch: dänische Stadt], Tallinn, Pelgulinn [deutsch wörtlich: Schutzstadt] bergen in sich diese gehoffte oder erhoffte Feste. Diese ursprüngliche Bedeutung verlautet deutlich auch in der Strophe eines Liedes von Martin Luther: Eine feste Stadt und Zufluchtsort ..., die angeblich zum ersten Mal 1529 in Wittenberg im Druck erschienen ist - auf Deutsch ursprünglich Ein' feste Burg ist unser Gott...

Auf überraschender Weise wird hier die Stadt - auf Deutsch bedeutet "die Burg" eine Festung, eine Schanze, ein befestigtes Schloss - ... mit Gott im Himmel verglichen, der an sich meiner Meinung nach keiner Vergleiche bedürfte. Und diese eine - Eine feste Stadt... - steht hier nicht in der Bedeutung irgendeiner beliebiger, sondern in der ursprünglichen festen grammatischen Funktion, die damals ins Estnische mitübersetzt wurde, die aber inhaltlich auch zugunsten des (einen) einzigen „unseren Gottes“ ausgelegt werden könnte - indem dabei doch eine gewisse Unsicherheit der oberirdischen Städte betont wird. Oder handelt es sich bei diesem Vergleich tatsächlich um den Mächtigsten, um folglich nur die mögliche Mächtigkeit der oberirdischen Stadt zu betonen? - Ich als Lehrer (der Philosophie) - auch im gegebenen Moment befinden wir uns in einer (Herbst)Schule - habe auch früher beständig über die Natur der Sachen nachdenken müssen, mir scheint, dass in einem Sinne, in einem tief verborgenen Sinne die Natur der Stadt der tiefsten Natur des Staates ähneln muss, was ich mir bereits seit langem klar gemacht hatte, was aber meistens hinter den „äußeren“ Definitionen abhandenkommt. Die Natur der Stadt - wie auch des Staates - würde nämlich darin bestehen, dass die Beziehung sowohl des Staates als auch der Stadt zu ihren Menschen wie das Verhältnis zwischen einem Kind und seiner Mutter

8 Siehe Eesti etümoloogia-sõnaraamat, 242. 
wäre - so wie die Mutter, indem sie ihr Kind auf ihren Schoß nimmt, es seiner Bewegungen gleichzeitig sowohl einschränkt als es dabei auch behütet, beschützt und beschirmt, ebenso sollte es auch im Falle der Stadt sein, die im heutigen Sinn der Begriffe historisch vor einem Staat entstand - erst die Städte werden zu Staaten... - denken wir dabei zum Beispiel an die polis des alten Griechenlands, zu deren späterer erläuternden Entsprechung der Stadtstaat wurde. Diese genannte Beziehung wäre demnach gegenseitig, wenn Sie so wollen, und dann im weitesten Sinne eine Beziehung der Achtung und Liebe. Das Verhältnis der Stadt zum Stadtbürger, dem Städter, wäre am nächsten im Sinne des Behütens, Beschützens und Beschirmens als auch im Sinne der Einschränkung, aber das gleiche - nicht nur das Beschützen, sondern auch die Einschränkung, „das bei der Stange Halten“ - sollten nicht nur die Städter seitens der Stadt, sondern auch die Stadt seitens der Städter ertragen. So wie auch das Kind die Mutter sowohl einschränkt als auch behütet. - Hier sage ich, während ich ein bisschen vom Thema abweiche, dass die Stadt Tartu meiner Meinung nach über ein solches Verhältnis mit dem Stadtbürger Tiit Veeber verfügte, der sich bekanntlich öffentlich auch so nannte. - Und wenn weiter oben die Rede von der Feindseligkeit der Stadt gegenüber dem wohlwollenden, gutgläubigen in der Stadt Angekommenen oder Ankommenden war, dann bedeutet das den Verlust der ursprünglichen Natur der Stadt, die Verwandlung der Stadt in etwas anderes, obzwar einige äußere formale Merkmale der Stadt erhalten bleiben, als ob weiterhin alles in Ordnung ist, aber dennoch nicht ist ... Wie dies oftmals auch mit einem Staat passieren kann. - Sowohl ich als vielleicht auch manch ein anderer wusste nicht genau, was man mit der besonderen Bezeichnung der Herbstschule „Diese Stadt und eine andere“ gemeint haben könnte. Von ihrem Aufbau her assoziiert sie offensichtlich mit der Überschrift eines der frühesten Theaterstücke von Mati Unt, „See maailm või teine“ [deutsch: „Diese Welt oder die andere"] (1966). Jedenfalls kann unsere Bezeichnung auf sehr unterschiedliche Weise verstanden werden. Und während ich über die mögliche Feindseligkeit der Stadt nachdachte, erinnerte ich mich sofort an eine andere Stadt, nämlich Moskau, die zum Beispiel in der russischen Filmkunst in der letzten Zeit in dem Sinne reichlich ausgebeutet worden ist: ein in Moskau angekommenes Mädchen vom Dorf- oder aus der Provinz findet in dieser Stadt - diese Stadt oder eine andere - etwas völlig anderes vor, als es gehofft hatte, sich jemals vorgestellt oder geträumt hatte ... Als Vorbild 
diente natürlich das klassische sowjetische Melodrama, das eigentlich unnachahmliche „Moskau glaubt den Tränen nicht" (russisch: Moskva slezam ne verit, 1979).

Am Anfang hatte ich vor, gründlicher natürlich über Tartu zu sprechen - und nur über Tartu. Genau in jener Art und Weise, die ich für die einzig mögliche hielt - wie auch der Tartuer Oskar Luts in seinem oben erwähnten Erinnerungswerk mit dem „binär-oppositionellen“ Titel „Maa ja linn“ [deutsch: „Land und Stadt"] (1939). Dort erzählt er zum Beginn folgendes: „Natürlich ist es so, wie jemand die Sache sieht. - an „mein“ altes Tartu erinnert sich wahrscheinlich noch jeder Einwohner dieser Stadt, der ein paar Jahre über das mittlere Alter hinausgekommen ist, etwa vor 45 bis 50 Jahren, als der botanische Garten noch Krongarten genannt wurde und so weiter." - Und weiter fragt "der dahingeschiedene Mummelgreis", d.h. der Vater, den sagen wir sieben Jahre alten Andres aus Alamuse: "Ich fahre morgen in die Stadt - willst du mitkommen?" - „Die Stadt“ - dabei handelt es sich gemäß des Verständnisses der damaligen Leute aus Alamuse einzig um Tartu. In dieser Gegend gibt es auch solche Personen, die Tallinn, Narva oder sogar St. Petersburg besucht haben, aber die sind, insbesondere nach dem Wissen der jüngeren Bevölkerung, als wären sie gar keine Städte, sie sind Gott weiß was ... Jedenfalls solche Orte, über die man sich nicht den Kopf zerbrechen sollte. Unsere Stadt ist einzig - Tartu, und dorthin macht es von Alamuse etwa vierzig Werst." ${ }^{\prime 9}$ Als ich das noch einmal wiedergelesen hatte, antwortete ich auch selbst auf die Frage vom „Mummelgreis“: ,....willst du mitkommen?“ ohne zu zweifeln: „Natürlich will ich!“ Ich machte dann mit dem altem Tartu (ungefähr im Jahre 1893) an der Hand von Andres aus Alamuse erneut Bekanntschaft und bedauerte das nicht, obwohl ich, wie gesagt, am Anfang vorgehabt hatte, mir dieses Tartu selbst auf irgendeine Art und Weise vorzunehmen - so wie ich es lange Zeit später, aber doch über Jahrzehnte „erlebt" habe. Da ich auch anderswo über einen längeren Zeitraum studiert und gelebt habe, das heißt nicht nur als Tourist, zum Beispiel in verschiedenen Städten Deutschlands und sogar in Moskau, halte ich mich dennoch für einen genuinen Tartuer - ich bin hier auf dem Domberg geboren worden, habe in ungefähr einem Dutzend Stadtteilen oder Straßen gelebt, auch in solchen verschiedenen Städten

9 Oskar Luts, Maa ja linn (Kaks muistset lugu) (Tartu: Noor-Eesti Kirjastus, 1939), 7-8. Ein Werst entspricht 1.066 Metern. Anmerkung des Übersetzers. 
wie Tammelinn [deutsch: Eichenstadt], Supilinn [deutsch: Suppenstadt] oder Annelinn [deutsch: Annestadt]. ${ }^{10}$ Keine von ihnen ist mir fremd. Ich selbst bin kein in die Stadt Gekommener oder Kommender, von denen weiter oben die Rede war, ich fand mich mal einfach im Haus Nr. 13 der Raudtee Straße in Tartu vor und habe deshalb die Stadt als ein Ankömmling ebenso wie ihre mögliche Feindseligkeit nicht antreffen können. Dieses Syndrom fehlt bei mir. Auch die Wohnortwechsel, diese Wanderung innerhalb von Tartu, haben keine deprimierenden Spuren hinterlassen. Als Stadt ist Tartu für mich tatsächlich die passendste, wesenseigene Stadt gewesen. - Ich glaube, dass wir alle manchmal darüber nachdenken sollten, wie unser Verhältnis zu der Stadt (oder den Städten) aussieht, in welcher (welchen) wir uns aufhalten ... Für mich ist dieses Tartu der ständige Wohnort gewesen, wie diese Bestimmung wenigstens in den frühen biographischen Lexika bei den Angaben zu einer öffentlichen Person verwendet wurde. Die Stadt Tartu hat mir Aufenthalt geboten.

Ich glaube, dass für mich dieses metaphorische, von einer Seite her beschützende, behütende und beschirmende Verhältnis und auf der anderen Seite auch ein Verhältnis der Einschränkung in Bezug auf Tartu auf Gegenseitigkeit beruhte. Während ich innerhalb von Jahrzehnten diese Stadt beobachtet habe - dabei wurde innerhalb der letzten zwei Jahrzehnte das Fahrrad zum besten Mittel hierfür - habe ich als einen Maßstab „die Sicht" meines verstorbenen Vaters eingesetzt, der diese Stadt fast die letzten fünfzig Jahre nicht mehr gesehen hat ... dass was er noch sah in dem sich verändernden Tartu und was er nicht mehr sah. Ich gebe mit Hilfe von ein paar Beispielen darauf Acht, was ihn noch nicht einschränkte, mich aber später bereits eingeschränkt hat. Es gibt Einschränkungen, die ich verstehe, aber es gibt auch andere ... Dort, auf einem "Radiantweg“ der Nachkriegszeit, der den Rand des BarclayPlatzes auf der Uferseite des Emajõgi entlang von Ülikooli Str. 1 bis zur Kreuzung der Straßen Ülikooli und Vanemuise verlief und von wo aus sich eine freie, gute und weitläufige Aussicht in Richtung auf den Riiamägi [deutsch: Rigaer Berg] eröffnete und den ich als Schuljunge wenigstens ein paar Mal die Woche entlangging, um aus der Bibliothek für Kinder- und Jugendliche Bücher auszuleihen, steht nun alles absperrend, die Bewegung einschränkend, das ehemalige Gebäude der Post davor...

10 Es handelt sich um verschiedene Stadtteile von Tartu. Anmerkung des Übersetzers. 


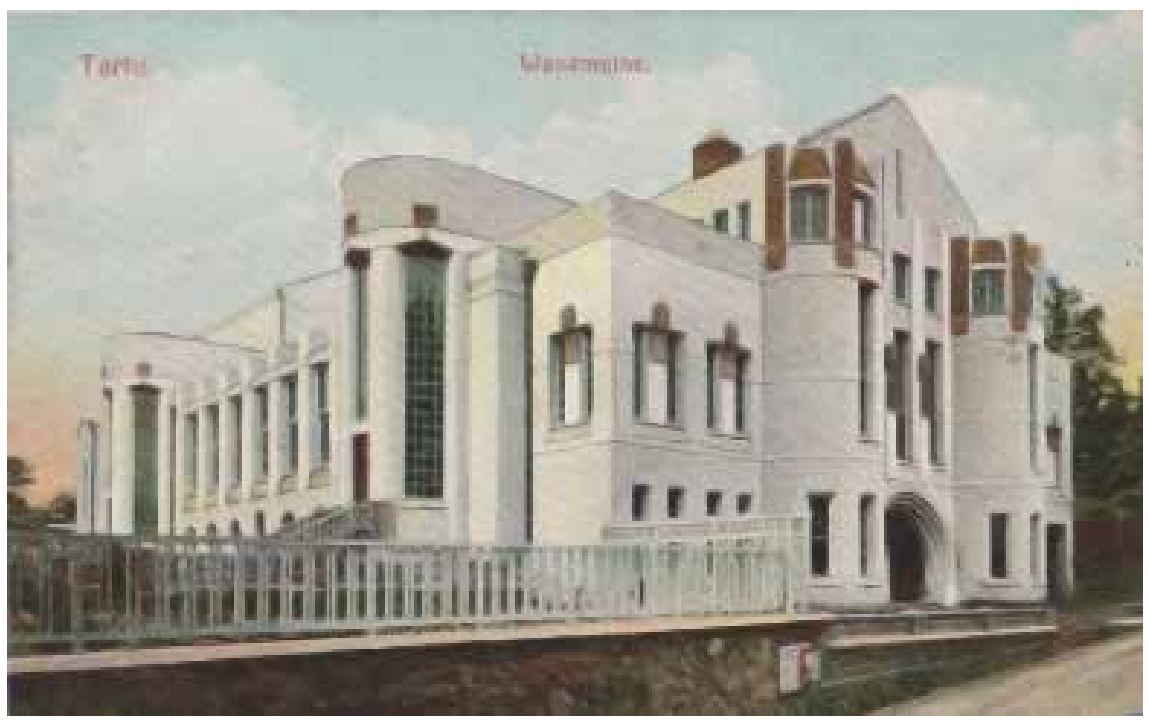

Abb. 1. Das Gebäude von Theater “Vanemuine” 1937/1939. Privatsammlung von Ants Linnard

Wer diesen Weg nicht gegangen ist, kann sich nicht daran erinnern, der kann kein solches Gefühl entwickeln, aber ich sehe darin in mehrerer Hinsicht eine Sackgasse ... Solche Beispiele könnte man noch mehr anführen. - Weiterhin kann ich mich gut daran erinnern, wie von einer Seite die teilweise Zerstörung von Karlova ${ }^{11}$ anfing, die mir auch über lange Zeit Aufenthalt geboten hat, mich bewahrt und beschützt hat. Ich entsinne mich, wie die Plattenbauten Schritt für Schritt weiter vordrangen wie die Panzer in Stalingrad, indem sie alles Lebendige auf ihrem Weg zerstörten, sowohl Straßen, Häuser als auch Gärten ... Damit ging der Durchbruch der Turu Straße von der anderen Seite einher, ebendaher unten, von der Seite vom Emajõgi ... Zum Glück ging die Sowjetmacht zugrunde, ging selber unter, bevor sie es geschafft hätte, ihren - meiner Meinung nach - größten Feind, den Teil des Stadtteils Karlova in Tartu, der auf der Seite von Ropka lag, komplett zu vernichten. Usw. Schließlich überkam mich noch ein nostalgisch-utopischer Gedanke - ich selbst glaube, dass er nicht besonderes ketzerisch ist -, dass man auch in Tartu außer den Ruinen des Dombergs einige weitere hätte erhalten müssen, die während des letzten Krieges entstanden waren, die aber erbarmungslos nach dem Krieg als Ergebnis der Vernichtungsarbeit,

11 Ein Stadtteil von Tartu. Anmerkung des Übersetzers. 


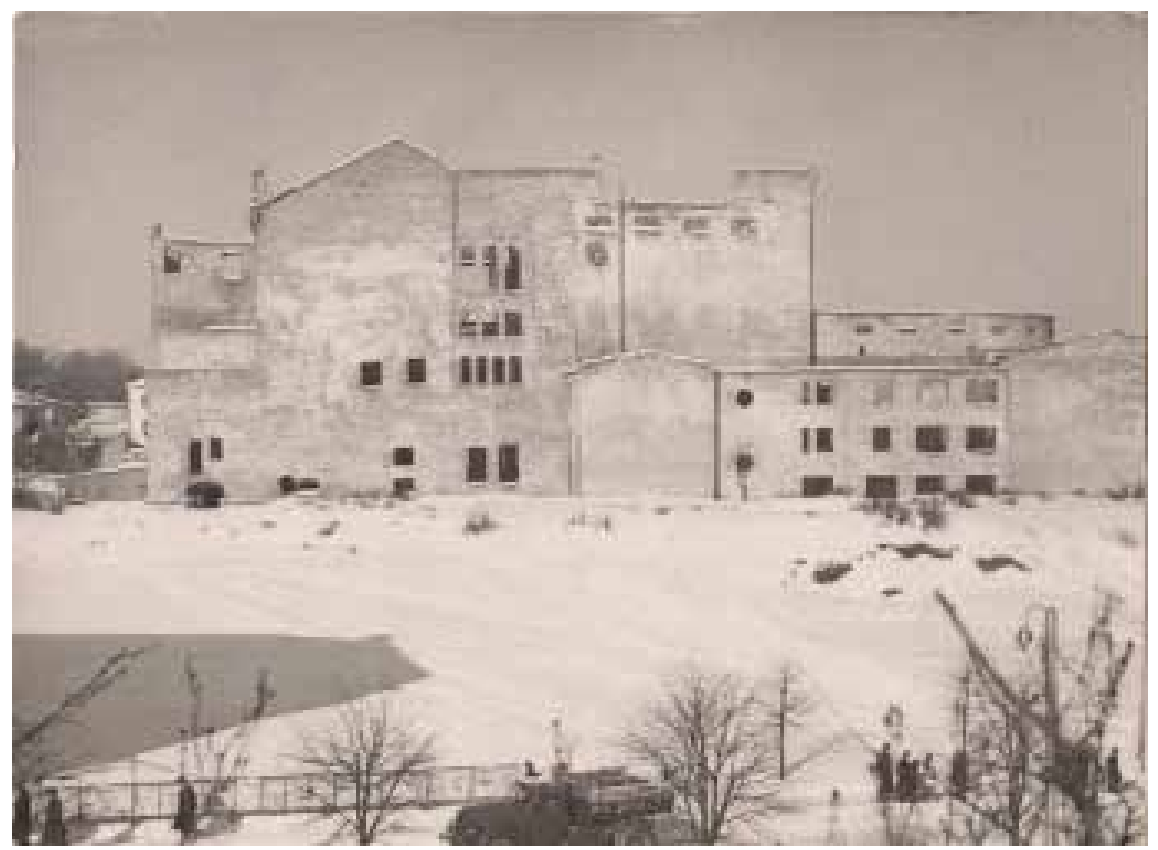

Abb. 2. Die Ruinen von Theater "Vanemuine" nach der Zerstörung 1944.

Privatsammlung von Ants Linnard

die unter der Bezeichnung "Aufbauarbeit" durchgeführt wurde, vernichtet wurden. Wenigstens in Europa hat man das gemacht, zum Beispiel stehen inmitten Berlins am Anfang des Kurfürstendamms die Ruinen der Gedächtniskirche, die zwar in das neue einheitliche Ensemble gehört, die man aber doch vor dem ursprünglichen Plan, die Kirchenruinen abzureißen, bewahren konnte. Ich meine hier im Falle von Tartu nicht den sofortigen Aufbau oder die Restaurierung einiger Bauten, sondern eher die Konservierung des Erhaltenen zum Zwecke des zukünftigen möglichen Wiederaufbaus. Was ich vor allem meine, ist das Theater- und Kulturhaus „Vanemuine“, das den Wiener geometrischen Jugendstil imitiert (1905-1906 Architekt Armas Lindgren, 1939 Architekt Arnold Matteus). Dass dies möglich gewesen wäre, bezeugt ein Bruchstück aus einem Interview von Jaan Malin mit dem Architekten Raul-Levroit Kivi (1995, ergänzt 1998 und 2003). Dort steht: „Wie sah sein [das Gebäude von „Vanemuine” - Ü.M.] Schicksal denn aus? Es blieb doch während des Feuergefechts zwischen den Fronten [1944] im Wege. Daran kann ich mich aber nicht erinnern, in welchem Jahr man mit der 


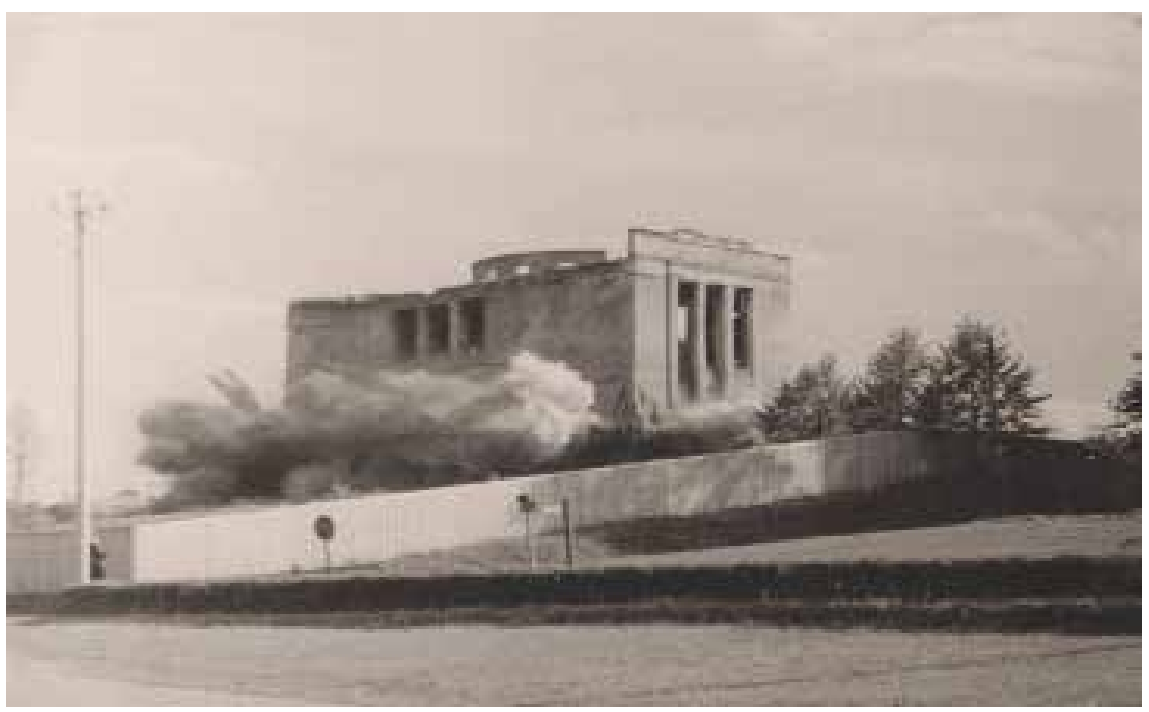

Abb. 3. Die Ruinen von Theater "Vanemuine" während der 1950er Jahren.

Privatsammlung von Ants Linnard

Durchführung der technischen[,] der Projektierung vorangehenden technischen Überwachung der Ruinen von der Vanemuine anfing, um ein Urteil über die Wiederaufbaufähigkeit zu liefern. Die Ruinen von Vanemuine waren ungefähr zehn Jahre lang der Witterung ausgesetzt. Die Ingenieure aus Tallinn stellten jedenfalls ein Dokument aus, laut dem die Ruinen durch die Witterung soweit beschädigt sind, dass man sie nicht einsetzen kann. Gemäß Matteus war so ein Dokument vorhanden. Ich weiß es zwar nicht sicher, dass es einen politischen Druck gegeben habe, dass das Jugendstil-Vanemuine nicht wiederaufgebaut werden darf und auf der Stelle dieser Ruinen etwas Modernes gemacht werden soll. [meine Betonung. - Ü.M.] Es kam einfach eine Ausschreibung, vom Jugendstil-Vanemuine blieb nichts übrig. / Die Frage besteht darin, warum die Ruinen nicht geschützt wurden. Nach dem Krieg war man wohl nicht in der Lage, irgendetwas zu schützen. Es gab eine panische Situation, in der man nicht wusste, von welcher Seite man anfangen sollte: in Ruinen standen die Johanniskirche, eine enorme Anzahl an Wohnhäusern usw."12 Warum so lange darüber berichten? Darum, weil ich fast jeden Tag, wenn ich das neue Gebäude passiere, das auf dem

12 Jaan Malin, „Intervjuu Raul-Levroit Kiviga“, Raul-Levroit Kivi, Tartu planeerimisest ja arhitektuurist: Artikleid ja mälestusi (Eesti Arhitektuurimuuseum: Tallinn, 2005), 93. 


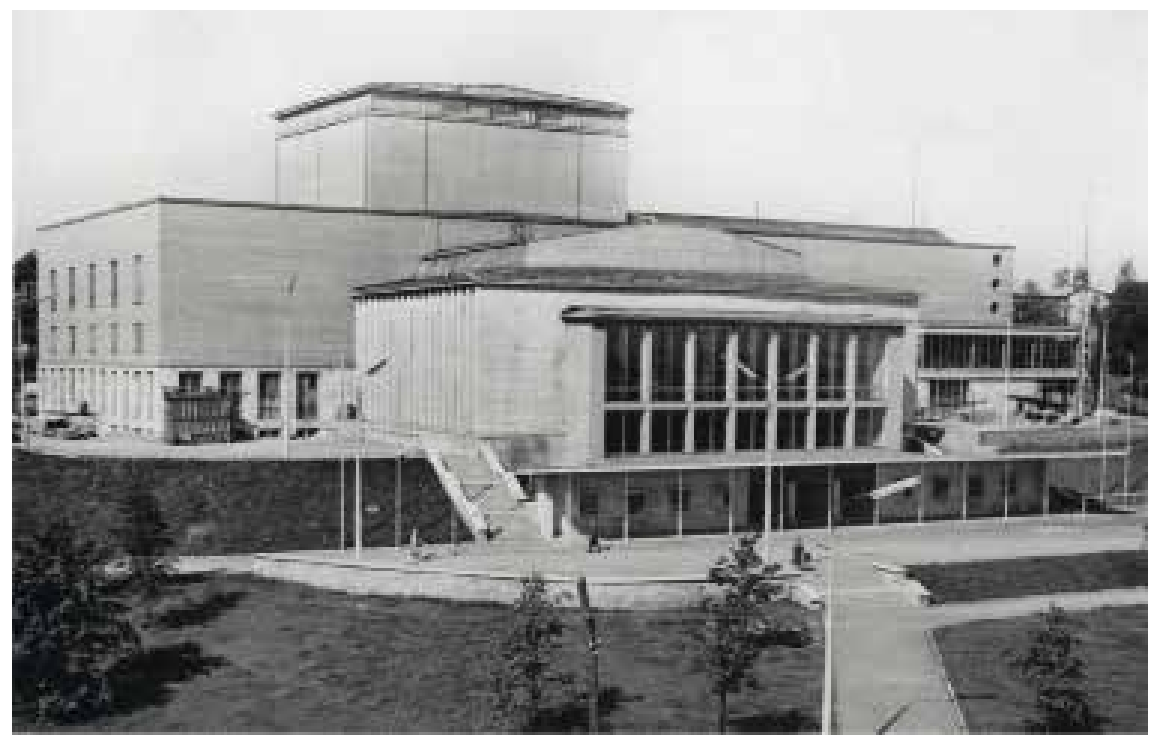

Abb. 3. Das neue Gebäude von Theater “Vanemuine” (eröffnet 1967).

Privatsammlung von Ants Linnard

Hügel in der Innenstadt steht, so etwas wie Gewissensbisse empfinde, denn als Junge der sechsten Klasse war ich einer von denjenigen, die dazu beitrugen, den Platz für das neue Gebäude von den Ruinen zu befreien, indem wir Steine und Bauschutt mit Hilfe einer Tragbahre weiter weg trugen. O sancta simplicitas! habe Jan Hus vom Scheiterhaufen einer Alten oder einem Bauern zugerufen, während er sah, wie sie oder er auch ihren/seinen dürren Baumzweig in die Flammen warf ... Aber Vanemuine selbst, der sich auf irgendeiner Weise hätte auch wundern können, zum Beispiel indem er vom Rande des Dombergs oder einer Wolke durch seinen Kannelspiel ${ }^{13}$ auf die falsche Tat Aufmerksam gemacht hätte, stand damals nicht zur Stelle. Es gab niemanden, der gerufen hätte. Jedenfalls fühle ich im Nachhinein, dass obwohl ich damals als Kind nicht begriff, was ich tat, das heißt meine Pflicht für die Stadt nicht erfüllte, sie nicht bewahren konnte, und die Stadt Tartu sich dafür bei mir nicht gerächt hat, obwohl - ehrlich gesagt - ich das neue Haus von „Vanemuine“ ziemlich selten besuche...

13 Beim Kannel handelt es sich um ein estnisches Saiteninstrument, das einer Zither ähnelt. Vanemuine ist der Gott der Musik gemäß des estnischen Nationalepos Kalevipoeg. Anmerkung des Übersetzers. 
Ich beende meine „Lehre“ mit einem Fragment von Bernard Kangro, der zwar nach Tartu aus Valga gekommen war, aber der für immer und ewig ein Tartuer blieb, wie er mir aus dem Gedächtnis in einem sehr lange zurückliegenden privaten Brief (aus Lund im Jahr 1968) zugestanden hat ... In dem ersten Roman seines Tartuer-Zyklus, ,Jäälätted" [deutsch: „Eisquellen“] sagt er folgendes: „Dein Schicksal ist Tartu, diese Stadt der Sehnsüchte, wo du versucht hast, um jeden Preis zu bleiben und für die du vieles geopfert hast. Du kannst dich nie hier losreißen, obwohl du es möchtest. Aber vielleicht ist dieses Tartu nach zehn Jahren gar nicht mehr das, was es damals war, als du das erste Mal hierhin kamst. Tartu verändert sich, aber du veränderst dich nicht. Oder umgekehrt - Tartu bleibt, aber du bist nicht mehr der alte. Tartu ändert sich mit jedem Jahr, du merkst das nur nicht. Es hat sich bereits verändert seitdem du kamst ..."14 - Bernard Kangro, der gar nicht mehr nach Tartu („hinein“) zurück konnte, sprach dennoch über sein Tartu paradoxerweise am genauesten: „Du kannst dich nie hier losreißen, obwohl du es möchtest." Während diese Stadt uns beschützt und bewahrt und beschirmt, schränkt sie uns ein, wie jede andere auch die seinen ... Es handelt sich um eine gutartige Einschränkung, fast um eine Art Liebeserklärung. Aber das gilt nur für die Städte, die der Natur der Stadt entsprechen ... Das hier ist eine Stelle zum Denken und zum Nachdenken. Für Bernard Kangro blieb Tartu für immer eine Stadt der Sehnsüchte - eine Stadt wie eine Erinnerung. Vielleicht war das die beste Lösung.

Wahrscheinlich hat man bemerkt, dass ich keine modernen Theorien oder Konzeptionen, -ismen oder -logien, wie das öfters in Mode ist, einsetzte - zum Beispiel, um zu sehen, dass ob und wenn, dann wie sich die "stadtauffassende“ alte Geschichte von Oskar Luts den neueren Errungenschaften der Narratologie anpasst ... Ich versuchte vor allen Dingen und nur die Worte und die Stadt selbst sprechen lassen, versuchte in erster Linie sie selbst zu vernehmen, um erst danach das Gesagte und das Gehörte mit dem von anderen wissenschaftlich Ausgesprochenen zu vergleichen, was von mir hier und heute jedoch unterlassen bleibt. Ich entschuldige mich dafür und bedanke mich beim Leser dafür, dass er mitunter auch mich einfach vernimmt.

14 Bernard Kangro, Jäälätted (Lund: Eesti Kirjanike Kooperatiiv, 1958), 50-51; Erneute Auflage (Tallinn: „Eesti Raamat”, 1990), 44-45. 
Ülo Matjus: The City as Memory: Untimely Notes

KeYWORDS: ESSENCE OF CITY, CITY AND TARTU IN ESTONIAN LITERATURE, CITY AND CITIZENRY, TARTU, HERITAGE PROTECTION

\section{SUMMARY:}

The essay focuses on the city and its essence in the context of memory. It is asserted that the true essence of a city as such cannot be completely revealed by individual sciences or by explanatory dictionaries. Therefore, 1) the Estonian word linn (city) is allowed to reveal itself with reference to its origin, and 2) the city itself is allowed to show itself as it really is, i.e. phenomenologically. Essentially, the essence of a city consists of both caring for and restricting its own residents or citizenry and the essence of the citizenry is to care for and restrict its own city. Figuratively, the relationship between a city and its citizens can be compared to that of a mother and child. Examples of this correlation, and the possibility that the correlation can change to reciprocal animosity or, instead, to love, are cited from Estonian literature (Karl Ristikivi, Oskar Luts, Bernard Kangro and others). Based on memories, the author focuses on the university town of Tartu, the city of his birth and his hometown. The city not only suffered damage during World War II, but also in the post-war years. For instance, the Soviet city authorities carelessly blew up and demolished the ruins of the Vanemuine Theatre, built in the geometric Art Nouveau style of Vienna, instead of rebuilding it with the aim of conserving it for the future. Imminent total destruction also threatened the district that provided the author shelter during his childhood and adolescent years. Luckily Soviet power itself was destroyed - ended before it was able to totally destroy one of its "greatest enemies" (as the author sees it) - the section of Tartu's Karlova district adjacent to Ropka.

\section{CV:}

Ülo Matjus is a philosopher, philologist, bibliophil, professor of history of Estonian philosophy at the University of Tartu. His main research fields include phenomenological philosophy (in the broadest sense) and being-historical thinking in Germany; scientific (commented) translation of phenomenological philosophy and being-historical thinking to Estonian; historically specific characters of mental social phenomena 
(metaphysics, philosophy, science, morals, religion, values etc.) in front of their modern unitedness; appearance of the historically specific character of aesthetical phenomena - especially the art - in age of the modern technology; history of Estonian book and the bibliophily. 